\title{
Effect of a Vasopressin V2 Receptor Antagonist on Polycystic Kidney Disease Development in a Rat Model
}

\author{
Xiaofang Wang ${ }^{\mathrm{a}}$ Megan M. Constans ${ }^{\mathrm{a}}$ Fouad T. Chebib ${ }^{\mathrm{a}}$ Vicente E. Torres ${ }^{\mathrm{a}}$ \\ Lorenzo Pellegrini ${ }^{\mathrm{b}}$ \\ aDepartment of Nephrology, Mayo Clinic, Rochester, MN, USA; 'b Palladio Biosciences, Inc., Newtown, PA, USA
}

\section{Keywords}

Polycystic kidney disease $\cdot$ Vasopressin V2 receptor antagonist · Lixivaptan $\cdot$ PCK rat model

\begin{abstract}
Background: Vasopressin V2 receptor inhibition is a clinically validated mechanism of action in the treatment of autosomal dominant polycystic kidney disease (ADPKD). In this study, the effect of lixivaptan, a potent, selective vasopressin V2 antagonist, was evaluated in PCK rats, a validated animal model of PKD. Methods: Four-week old PCK rats were fed rodent chow with $0.5 \%$ lixivaptan (low dose) or $1 \%$ lixivaptan (high dose), or chow only (control) for 8 weeks. Urine output was measured at weeks 7 and 10 of age. Animals were killed at 12 weeks of age; kidneys and livers were collected, weighted, and analyzed for cyclic adenosine $3^{\prime}, 5^{\prime}$-monophosphate (cAMP) levels and cystic burden and fibrosis; serum creatinine and sodium were measured. Results: Consistent with the development of a polycystic kidney phenotype, control PCK rats showed enlarged kidneys, extensive cyst formation, and early signs of serum creatinine elevation at 12 weeks of age. Compared to controls, PCK rats treated with low-dose lixivaptan showed a $26 \%$ reduction in $\%$ kidney weight/body weight $(p<0.01) ;$ a $54 \%$ reduction in kidney cystic score ( $p<$ 0.001 ), a histomorphometric measure of cystic burden; a
\end{abstract}

(c) 2019 S. Karger AG, Basel

E-Mail karger@karger.com

www.karger.com/ajn
$23 \%$ reduction in kidney cAMP levels $(p<0.05)$, a biochemical marker of disease; and a $13 \%$ reduction in plasma creatinine $(p<0.001)$, indicating preserved renal function. These reductions were associated with 3 -fold increases in 24-h urine output, demonstrating the potent aquaretic effect of lixivaptan. The fact that the high dose was less efficacious than the low dose is discussed. Conclusions: These results provide the first evidence of the potential utility of lixivaptan for the treatment of ADPKD.

(c) 2019 S. Karger AG, Basel

\section{Introduction}

Autosomal dominant polycystic kidney disease (AD$\mathrm{PKD}$ ) is an inherited genetic disease characterized by the formation and enlargement of fluid-filled cysts in the kidneys, liver, and other organs, resulting in progressive loss of renal function [1]. ADPKD results from loss-of-function mutations in polycystin (PKD) 1 or 2 genes, which disrupt the normal differentiated phenotype of the renal tubular epithelium, leading to increases in intracellular cyclic adenosine $3^{\prime}, 5^{\prime}$-monophosphate (cAMP), and resulting in increased cellular proliferation and cyst formation [2]. Cyst growth displaces and damages normal kidney tissue, leading to a decrease in nephron function. 
ADPKD is one of the most common genetic diseases, with an estimated incidence as high as 1 per $500-1,000$ [1]. It is estimated to affect 12.5 million people worldwide. ADPKD accounts for $5-10 \%$ of end-stage renal disease cases, with approximately $50 \%$ of patients with ADPKD developing end-stage renal disease by the age of 60. This makes ADPKD the fourth leading cause of kidney failure.

A large body of preclinical and clinical data have established vasopressin V2 receptor antagonism as a clinically validated mechanism of action in the treatment of ADPKD [3]. In particular, the vasopressin receptor antagonist tolvaptan proved efficacious in 2 large phase 3 clinical studies in ADPKD $[4,5]$ and is currently approved in the United States, Europe, Japan, and Canada for this indication in patients with rapidly progressive disease. In the United States, the US Food and Drug Administration has determined that tolvaptan should be used only under a strict risk management program because it can cause liver injury, potentially requiring liver transplant or leading to death [6]. Therefore, despite these advances, an effective disease-modifying therapy for the treatment of ADPKD without potential liver toxicity is still lacking. Lixivaptan is a potent, selective vasopressin V2 receptor antagonist that has shown efficacy in the correction of hyponatremia associated with syndrome of inappropriate antidiuretic hormone secretion, heart failure, and liver cirrhosis with ascites, with few adverse effects reported [7]. Importantly, preliminary in vitro and in silico evidence suggests that lixivaptan is less likely to cause hepatotoxicity than tolvaptan at their respective therapeutic doses for the treatment of ADPKD [8], suggesting that lixivaptan could provide a safer alternative to tolvaptan for the treatment of ADPKD.

The PCK rat model is an orthologous model of human PKD caused by a splicing mutation in the $P k h d 1$ gene and is a well-established and extensively used model in the field of PKD research $[9,10]$. It is characterized by progressive cystogenesis, increased levels of renal cAMP, and impairment of renal function that are phenotypically similar to human ADPKD. Importantly, the vasopressin receptor antagonist tolvaptan was found to be effective in the PCK rat model [11], validating the model as a possible predictor of efficacy in humans.

The goal of the present study was to determine the effects of lixivaptan in the PCK rat model with particular emphasis on disease manifestation endpoints (kidney weight as percentage of total body weight, cyst burden, renal cAMP levels, and serum creatinine) as well as pharmacodynamic endpoints (urine output and serum electrolyte concentration). In addition to kidney manifestations, the majority of patients with ADPKD also develop liver cysts and fibrosis; therefore, we also investigated whether lixivaptan could improve liver pathology in PCK rats.

\section{Materials and Methods}

\section{Animals}

The Institutional Animal Care and Utilization Committee approved all experimental protocols described herein. The PCK rats (a Sprague-Dawley strain) used in this study are maintained in the animal facilities of the Department of Veterinary Medicine of the Mayo Clinic (Rochester, MN, USA). The animals were housed 2 males or 3 females per cage and fed ground rat chow at libitum (Purina Mills, Richmond, IN, USA).

\section{Study Design and Procedures}

At 4 weeks of age, PCK rats were divided into 3 groups: one control group and 2 treatment groups of 20 animals each (10 females and 10 males). Animals received either ground rodent chow containing $0.5 \%$ lixivaptan (low-dose group), $1 \%$ lixivaptan (highdose group), or rodent chow without lixivaptan (control group) for 8 weeks. Pharmacokinetic experiments have shown that these oral doses of lixivaptan yielded an exposure comparable to the administration of approximately $30-100 \mathrm{mg} / \mathrm{kg}$ lixivaptan by oral gavage (unpublished data). Diets were prepared by dissolving lixivaptan in DMSO at a concentration of $0.5 \mathrm{~g} / \mathrm{mL}$ and mixing the resulting lixivaptan solution with the solid diet (2016CM Harlan Teklad Global Rodent Diet ${ }^{\circledR}$, Harlan Teklad, Inc., Indianapolis, IN, USA) for $1 \mathrm{~h}$ using a high capacity food processor. Control group diet contained DMSO but no drug. All food preparations were made fresh weekly at the Product Safety Labs facility in Monroe, NJ, USA, and stored in the dark until ready for use. Food was provided based on $5 \mathrm{~g}$ of feed per $100 \mathrm{~g}$ of animal body weight. Food intake and animal weight were monitored weekly.

Animals were placed in metabolic cages at 7 and 10 weeks of age to measure 24-h urine outputs. At 12 weeks of age, animals were weighed and killed by anesthesia with intraperitoneal ketamine $(90 \mathrm{mg} / \mathrm{kg})$ and xylazine $(10 \mathrm{mg} / \mathrm{kg})$. Blood was collected by cardiac puncture for determination of plasma electrolytes, creatinine, and urea levels. The right kidney and part of the liver were placed into preweighed vials containing $10 \%$ formaldehyde in phosphate buffer $\mathrm{pH}$ 7.4. The tissues were embedded in paraffin for histological studies. The left kidneys were flash frozen in liquid nitrogen for determination of cAMP.

\section{Kidney and Liver Histomorphometric Analysis and Immunohistology}

Five-micron transverse tissue sections of kidney (including the cortex, medulla, and papilla) and liver were stained with hematoxylin-eosin, Masson's trichrome, and picrosirius red. Whole transverse tissue sections with hematoxylin-eosin staining were used to measure cyst volumes. Picrosirius red staining of collagen fibers was used for fibrosis analysis, and whole sections were used for hepatic fibrosis. All tissue sections were stained with picrosirius red in a single batch to avoid variability of staining from batch to batch. For renal fibrosis, sequential images were collected using the $10 \mathrm{x}$ objective, moving along the center line of each section 
Table 1. Effects of lixivaptan on various renal parameters

\begin{tabular}{|c|c|c|c|}
\hline Parameters & Control & Lixivaptan low dose, $\%$ & Lixivaptan high dose, $\%$ \\
\hline \multicolumn{4}{|l|}{ 24-h urine output, $\mathrm{mL}$} \\
\hline \multicolumn{4}{|l|}{ Week 7} \\
\hline$\%$ change vs. control & - & 175 & 223 \\
\hline \multicolumn{4}{|l|}{ Week 10} \\
\hline Mean \pm SD & $17.5 \pm 6.9$ & $50.6 \pm 15.5^{* * *}$ & $59 \pm 15.9^{* * *}$ \\
\hline \multicolumn{4}{|l|}{ Plasma sodium, mEq/L } \\
\hline Mean \pm SD & $143.6 \pm 3.5$ & $146.0 \pm 3.2^{*}$ & $144.8 \pm 2.7$ \\
\hline$\%$ change vs. control & - & 1.6 & 0.8 \\
\hline \multicolumn{4}{|c|}{ Serum markers of renal function } \\
\hline \multicolumn{4}{|c|}{ Plasma creatinine, $\mathrm{mg} / \mathrm{dL}$} \\
\hline Mean \pm SD & $0.73 \pm 0.06$ & $0.63 \pm 0.08^{* * *}$ & $0.68 \pm 0.05^{* *}$ \\
\hline \multicolumn{4}{|c|}{ Renal cAMP, $\mathrm{pmol} / \mathrm{mg}$ protein } \\
\hline Mean \pm SD & $20.5 \pm 6.4$ & $15.7 \pm 4.8^{*}$ & $23.8 \pm 9.8$ \\
\hline$\%$ change vs. control & - & -23 & 16 \\
\hline $\begin{array}{l}{ }^{*} p<0.05 . \\
{ }^{* *} p<0.01 . \\
* * * p<0.001 \text { vs. control. } \\
\text { cAMP, cyclic adenosine }\end{array}$ & & & \\
\hline
\end{tabular}

from one end of the available cortex to the other, without overlapping, in order to obtain an unbiased sampling. Renal medulla was not measured. For hepatic fibrosis, the whole sections were analyzed.

Analysis of light microscopy images was performed using the NIS-Elements AR software system (Nikon, Elgin, IL, USA), which includes a light microscope with a color digital camera (Nikon DS-Ri1) and a Pentium IBM-compatible computer (HP Z420). Stained sections were visualized under a Nikon microscope, and digital images were acquired using a high-resolution Nikon digital camera. The observer could interactively apply techniques of enhancement for a better definition of interested structures or exclude fields too damaged to be analyzed. Visually, veins and bile ducts were excluded. To calculate the volume of renal or hepatic cyst or fibrosis, a colored threshold was applied at a level that separated the objects from their background. The kidney/liver cyst and fibrosis scores were then expressed as a percentage of the area with cysts or fibrosis over the total area analyzed.

\section{cAMP Content of Whole Kidneys}

Frozen kidneys were weighed and ground to fine powder under liquid nitrogen using a stainless-steel mortar and homogenized in 10 volumes of $0.1 \mathrm{M} \mathrm{HCl}$ at room temperature. Total protein concentration was measured using the BCA Protein Assay Kit (Pierce, IL, USA). After centrifugation at $600 \times \mathrm{g}$ for $10 \mathrm{~min}$ at room temperature, the supernatant was further diluted in $0.1 \mathrm{M} \mathrm{HCl}$ and run directly in the CAMP assay or stored frozen for later analysis. The cAMP content was assessed by Direct cAMP ELISA kit according to the manufacturer's instructions (Enzo Life Sciences, Inc., Farmingdale, NY, USA). Samples were processed in triplicate. The results were expressed in $\mathrm{pmol} / \mathrm{mg}$ of protein.

\section{Other Analyses}

Blood collected at week 12 was used to measure plasma creatinine, urea, sodium, and potassium. Plasma creatinine and urea were measured using the Quantichrome creatinine and urea assay kits (Bioassay Systems, Hayward, CA, USA). Measurements of plasma sodium and potassium were performed using a $\mathrm{pHOx}$ Ultra Comprehensive Critical Care Analyzer (Nova Biomedical, Waltham, MA, USA).

\section{Statistical Analysis}

Data were expressed as means $\pm \mathrm{SD}$. Comparisons between groups were analyzed using 1- or 2-way analysis of variance with least significant difference comparisons of the means or Student $t$ test, as appropriate. All analyses were performed using the PRISM software (La Jolla, CA, USA). $p<0.05$ was considered a statistically significant difference.

\section{Results}

\section{Effect of Lixivaptan on Urine Output}

Treatment with lixivaptan resulted in approximately 3-fold increases in urine output at weeks 7 and 10 (Table 1). 


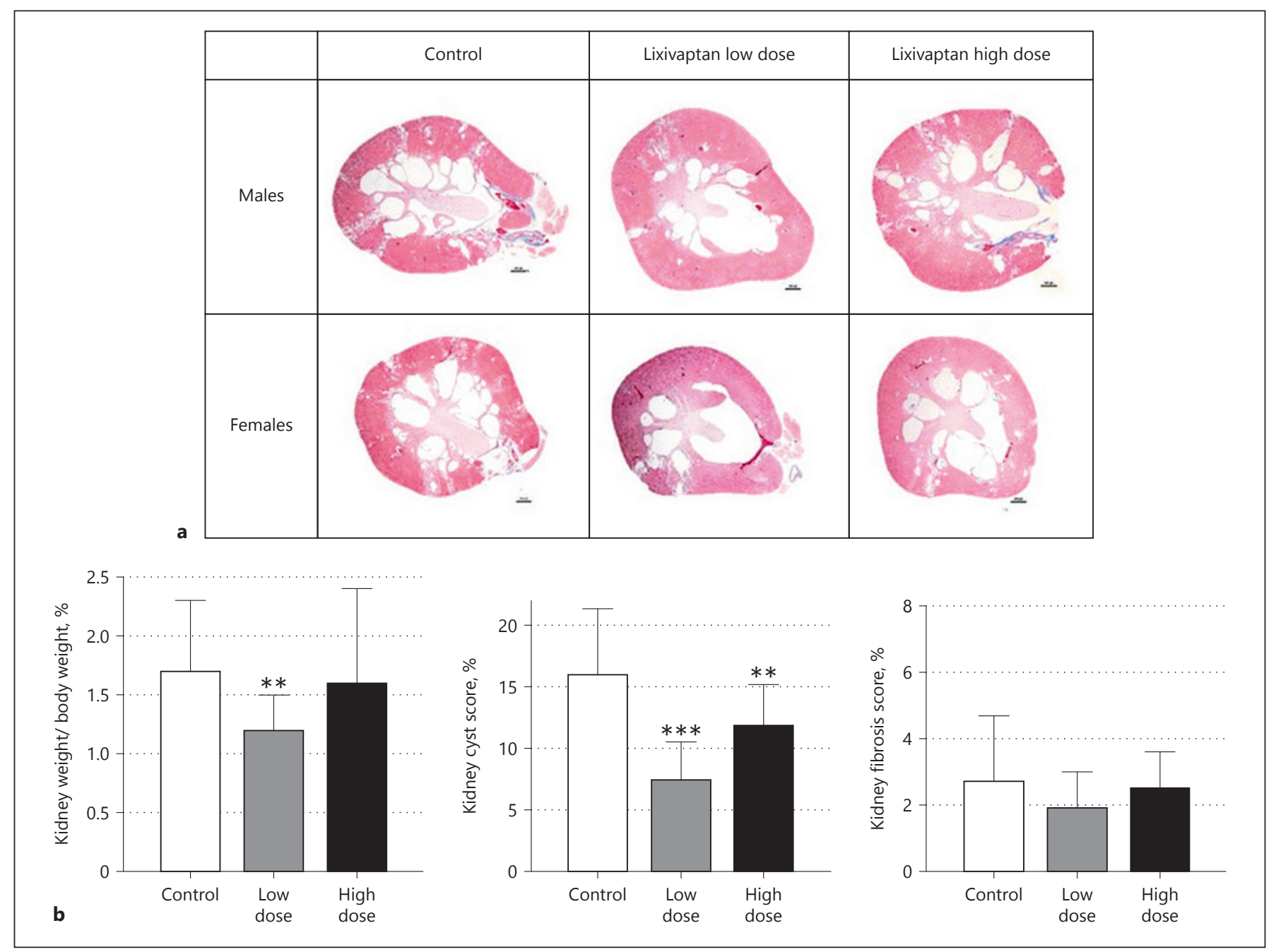

Fig. 1. Effect of lixivaptan on renal morphology. a Representative histological images of transverse kidney sections from PCK rats treated with lixivaptan compared with controls. b Effect of lixivap- tan on \% kidney weight/body weight, kidney cyst score, and kidney fibrosis score. Data are mean $\pm \mathrm{SD}$. ${ }^{* *} p<0.01,{ }^{* * *} p<0.001$ vs. control.
Plasma sodium was slightly increased in the low-dose group (Table 1), whereas plasma potassium was unchanged (data not shown). These effects are consistent with the potent aquaretic activity of lixivaptan and its associated increase in natremia [12].

\section{Effect on Renal Morphology}

Consistent with the development of a polycystic kidney phenotype, PCK rats in the control group showed enlarged kidneys (measured by \% kidney weight/body weight), extensive cyst formation (measured by the kidney cyst score), and significant kidney fibrosis (measured by the kidney fibrosis score). Treatment with low-dose lixivaptan was associated with a $26 \%$ reduction in relative kidney size $(p<$
$0.01)$, a $54 \%$ improvement in cyst score $(p<0.001)$, and a $30 \%$ decrease in kidney fibrosis ( $p=0.12$; Fig. 1). Highdose lixivaptan was associated with a $3 \%$ reduction in relative kidney size ( $p>0.05)$, a $25 \%$ improvement in cyst score $(p<0.01)$, and a $10 \%$ decrease in kidney fibrosis $(p>0.05$; Fig. 1). The statistical separation between the high-dose lixivaptan group and control group was negatively impacted by the presence of 3 outliers in the high-dose group that showed concomitant elevated ratios of organ weight versus body weight for kidney, heart, liver, and/or spleen. The significance of this finding is unknown. Removing these 3 outliers from the high-dose group and one similar outlier from the control group resulted in a significant difference between the high-dose group and control group for the $\%$ 


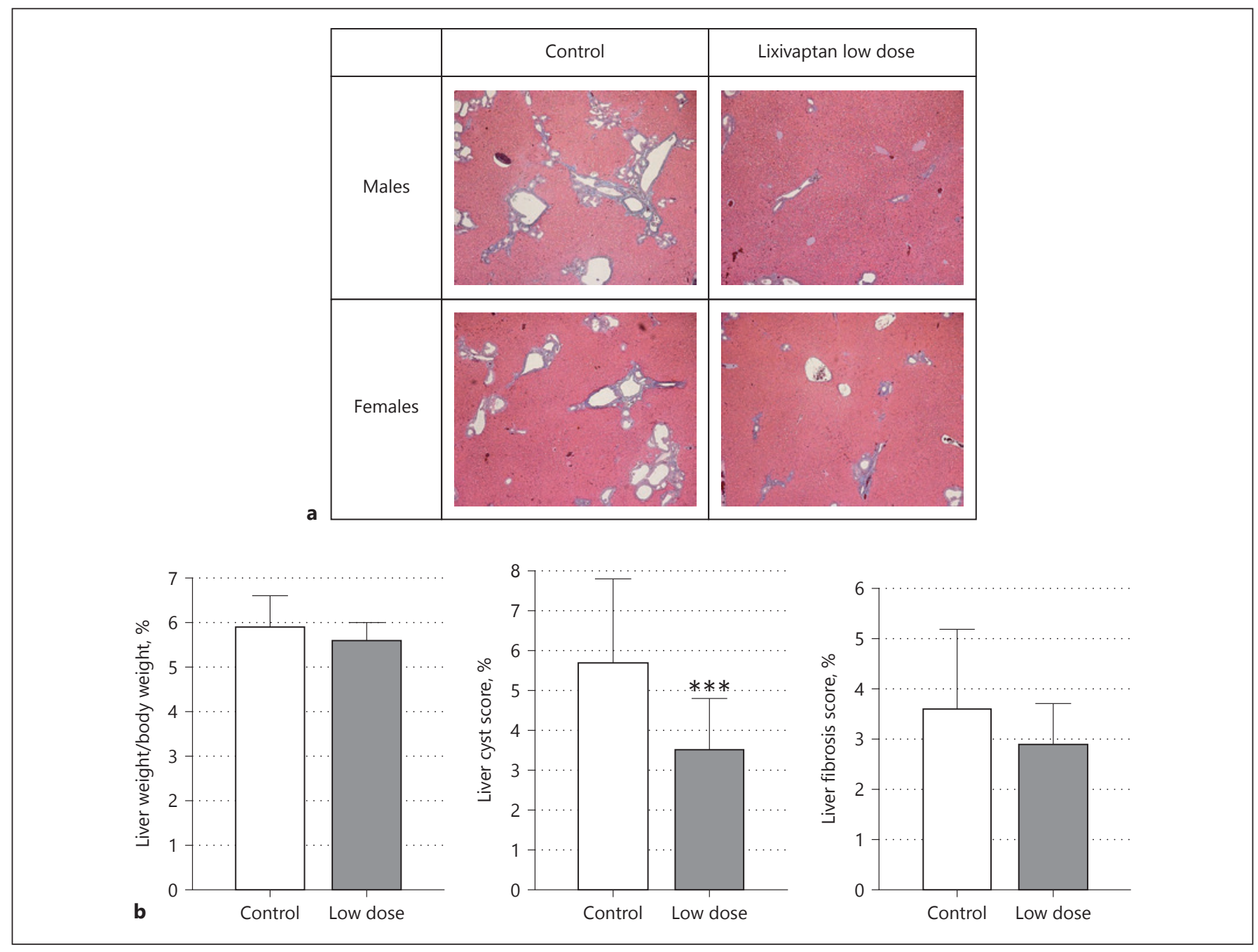

Fig. 2. Effect of lixivaptan on liver morphology. a Representative histological images of transverse liver sections from PCK rats treated with lixivaptan low dose compared with controls. b Effect of lixivaptan on \% liver weight/body weight, liver cyst score, and liver fibrosis score. Data are mean \pm SD. ${ }^{* * *} p<0.001$ vs. control. kidney weight/body weight (1.3 vs. $1.6 \%$, respectively; $p<$ 0.01 ; analysis presented in online suppl. Material, see www. karger.com/doi/10.1159/000500667).

\section{Effect on Markers of Renal Function}

Elevated creatinine and urea are hallmark of PKD and markers of renal function [1]. Compared to control animals, treatment with lixivaptan at either dose was associated with statistically significant reductions in serum creatinine (low-dose group: $-13.2 \%, p<0.001$; high-dose group: $-7.4 \%, p<0.01$; Table 1 ), suggesting a protective effect on renal function. Lixivaptan also reduced plasma urea by $16.1 \%$ in the low-dose group $(p=0.10)$ and $8.8 \%$ in the high-dose group $(p>0.05$; Table 1).

Effect of Lixivaptan in Polycystic Kidney Disease Rat Model
Elevated intracellular cAMP plays a pivotal role in the cellular processes that lead to the development of a PKD phenotype [13]. Accordingly, renal cAMP levels are elevated in PCK rats compared to wild-type animals, and a positive correlation exists between the tissue levels of cAMP and the severity of PKD [3]. Treatment of PCK rats with low-dose lixivaptan led to a $23 \%$ decrease in cAMP levels compared to control animals $(p<0.05)$; the highdose group did not separate from control (Table 1).

\section{Effect on Liver Parameters}

Treatment with low-dose lixivaptan also showed a protective effect on the development of disease manifestations in the liver (Fig. 2). A histomorphometric analy- 
sis was conducted to quantitate the treatment effect (Table 1). Compared to controls, animals treated with lowdose lixivaptan showed highly significant reductions in liver cyst score $(39.3 \%$ decrease; $p<0.001)$, accompanied by reductions in \% liver weight/body weight $(5.2 \%$ decrease; $p>0.05)$ and fibrosis score $(20.6 \%$ decrease; $p>0.05)$. Conversely, the high-dose lixivaptan group did not separate from control for liver parameters (data not shown).

\section{Safety}

The administration of lixivaptan was well tolerated over the study duration. All animals completed the experiment. Three animals ( 1 male and 2 females) treated with high-dose lixivaptan and one control animal showed concomitant elevated ratios of organ weight vs. body weight for kidney, heart, liver, and/or spleen. The cause of this finding is unknown and was not attributed to lixivaptan.

\section{Discussion}

This study represents the first assessment of lixivaptan's activity in a well-established animal model of PKD. Altogether, findings from this study indicated that lixivaptan was safe and well tolerated and improved kidney and liver disease manifestations in the rat PKD model. Lixivaptan also safely increased urine output and natremia, which is consistent with the effect observed with the vasopressin 2 receptor antagonist tolvaptan [14] and in previous lixivaptan studies conducted in human subjects [12].

First, a beneficial effect of lixivaptan was observed consistently across all aspects of renal disease, with lixivaptan reducing kidney size, cyst burden, fibrosis, and markers of renal function (cAMP levels and serum creatinine). Importantly, the magnitude of effect on kidney enlargement, cyst burden, and renal cAMP seen with low-dose lixivaptan in this study were all statistically significantly different from controls and were comparable to those observed with the vasopressin V2 receptor antagonist tolvaptan in the same PCK rat model. In addition, lixivaptan also exerted a highly statistically significant beneficial effect on plasma creatinine, a parameter not assessed in the Wang et al. [11] study. Taken together, these results provide the first direct evidence of lixivaptan's utility as a potential therapy for PKD.
An intriguing finding of the present study was the beneficial effect of lixivaptan on hepatic pathology. Indeed, animals treated with the low dose of lixivaptan showed a significant reduction in liver cyst burden and a nonsignificant effect on liver fibrosis. Because the vasopressin V2 receptor is expressed in cholangiocytes, where it directly modulates biliary epithelium growth [15], lixivaptan's beneficial effect on liver manifestations of disease could be explained by its ability to inhibit biliary epithelium growth. Of note, however, no effect on hepatic pathology was observed in the group treated with high-dose lixivaptan in this study. To our knowledge, this beneficial liver effect is unique to lixivaptan since no other vasopressin antagonists, including tolvaptan, have demonstrated any liver effect in the PCK rat model [3, $11,16]$. Because these other vasopressin antagonists are less selective than lixivaptan for the V2 vasopressin receptor relative to the V1 vasopressin receptor, and because the effect of lixivaptan was not seen at higher doses, one could speculate that the positive effect of lixivaptan on liver morphology may be counteracted by V1 receptor antagonism that occurs at these higher doses. Additional studies exploring wider dose ranges will be needed to conclusively prove a beneficial effect of lixivaptan on liver pathology.

Effects in the lixivaptan low-dose group were systematically greater than in the high-dose group, the latter only reaching statistical significance versus controls for the cyst burden and plasma creatinine endpoints. A similar inverse dose - response effect was noted with other agents in the PCK model, including PPAR $\gamma$ agonists [17] and tolvaptan [11]. With tolvaptan, it was speculated that higher doses of the compound could lead to antagonism for the vasopressin V1a receptor and inhibition of intracellular calcium release, thereby limiting its potential benefit at higher doses [11]. A similar phenomenon could contribute to the reduced effect seen with high doses of lixivaptan in the current study. An alternative explanation for the lower effectiveness of high-dose lixivaptan is the presence of a greater number of outliers in that group, as removal of these outliers from data analyses yields a statistical difference between the high-dose group and controls for kidney size. A third possibility is a reduced food intake by animals in the high-dose group (as demonstrated by their reduced weight gain compared to controls), suggesting reduced lixivaptan dosing. This proof-of-principle study was not intended to formally assess lixivaptan's dose - response relationship in the PCK model. An accurate dose - response assessment will require frequent 
test article administration by oral gavage using sex-specific doses.

In summary, the results of this study provide initial evidence to support the potential utility of lixivaptan as a promising new therapy for the treatment of PKD and pave the way for the demonstration of its efficacy in upcoming clinical trials.

\section{Acknowledgment} tance.

The authors thank Florence Paillard for her editorial assis-

\section{Statement of Ethics}

Animal experiments conform to internationally accepted standards and have been approved by the Institutional Animal Care and Utilization Committee of the Department of Veterinary Medicine of the Mayo Clinic (Rochester, MN, USA).

\section{Disclosure Statement}

V.E.T. has received research support from Otsuka and consulted for Vertex, Sanofi-Genzyme, and Palladio Biosciences. L.P. owns stock in Palladio Biosciences, Inc., which funded this study.

\section{Funding Sources}

This study was sponsored and funded by Palladio Biosciences, Inc. It was also supported in part by grants from the National Institutes of Health (DK44863 and DK90728) and by the Mayo Clinic Robert M. and Billie Kelley Pirnie Translational PKD Research Center.

\section{Author Contributions}

V.E.T.: study design, writing manuscript; F.T.C.: study design, executing experiments; X.W. and M.M.C.: executing experiments, collecting, and analyzing data; L.P.: study design, preparing tables and figures, writing manuscript.

\section{References}

1 Chebib FT, Torres VE. Autosomal dominant polycystic kidney disease: core curriculum 2016. Am J Kidney Dis. 2016 May;67(5):792810.

2 Saigusa T, Bell PD. Molecular pathways and therapies in autosomal-dominant polycystic kidney disease. Physiology (Bethesda). 2015 May;30(3):195-207.

3 Gattone VH 2nd, Wang X, Harris PC, Torres VE. Inhibition of renal cystic disease development and progression by a vasopressin $\mathrm{V} 2 \mathrm{re}$ ceptor antagonist. Nat Med. 2003 Oct;9(10): 1323-6.

4 Torres VE, Chapman AB, Devuyst O, Gansevoort RT, Grantham JJ, Higashihara E, et al.; TEMPO 3:4 Trial Investigators. Tolvaptan in patients with autosomal dominant polycystic kidney disease. N Engl J Med. 2012 Dec; 367(25):2407-18.

5 Torres VE, Chapman AB, Devuyst O, Gansevoort RT, Perrone RD, Koch G, et al.; REPRISE Trial Investigators. Tolvaptan in laterstage autosomal dominant polycystic kidney disease. N Engl J Med. 2017 Nov;377(20): 1930-42.

6 U.S. Food and Drug Administration [Internet]. JYNARQUE ${ }^{\circledR}$ (tolvaptan) tablets for oral use [Prescribing Information] [Revised: April 2018]. Available from: https://www.accessdata.fda.gov/drugsatfda_docs/ label/2018/204441lbl.pdf.
$7 \mathrm{Ku}$ E, Nobakht N, Campese VM. Lixivaptan: a novel vasopressin receptor antagonist. Expert Opin Investig Drugs. 2009 May; 18(5): 657-62.

8 Woodhead JL, Pellegrini L, Siler SQ, Shoda LK, Watkins PB, Howell BA. Prospective liver safety comparison of two treatments for autosomal-dominant polycystic kidney disease (ADPKD) using quantitative systems toxicology modeling [cited: April 1, 2019]. J Pharmacokinet Pharmacodyn. 44:S70-S71 [poster]. Available from: https://www.simulationsplus.com/assets/FINAL_Lixivaptan_DILIsym_ACoP_2017.pdf.

9 Katsuyama M, Masuyama T, Komura I, Hibino $\mathrm{T}$, Takahashi $\mathrm{H}$. Characterization of a novel polycystic kidney rat model with accompanying polycystic liver. Exp Anim. 2000 Jan;49(1):51-5.

10 Lager DJ, Qian Q, Bengal RJ, Ishibashi M, Torres VE. The pck rat: a new model that resembles human autosomal dominant polycystic kidney and liver disease. Kidney Int. 2001 Jan;59(1):126-36.

11 Wang X, Gattone V 2nd, Harris PC, Torres VE. Effectiveness of vasopressin V2 receptor antagonists OPC-31260 and OPC-41061 on polycystic kidney disease development in the PCK rat. J Am Soc Nephrol. 2005 Apr; 16(4): 846-51.
12 Abraham WT, Shamshirsaz AA, McFann K Oren RM, Schrier RW. Aquaretic effect of lixivaptan, an oral, non-peptide, selective V2 receptor vasopressin antagonist, in New York Heart Association functional class II and III chronic heart failure patients. J Am Coll Cardiol. 2006 Apr;47(8):1615-21.

13 Chebib FT, Sussman CR, Wang X, Harris PC, Torres VE. Vasopressin and disruption of calcium signalling in polycystic kidney disease. Nat Rev Nephrol. 2015 Aug;11(8):451-64.

14 Ohnishi A, Orita Y, Takagi N, Fujita T, Toyoki T, Ihara Y, et al. Aquaretic effect of a potent, orally active, nonpeptide V2 antagonist in men. J Pharmacol Exp Ther. 1995 Feb;272(2): 546-51.

15 Mancinelli R, Franchitto A, Glaser S, Vetuschi A, Venter J, Sferra R, et al. Vasopressin regulates the growth of the biliary epithelium in polycystic liver disease. Lab Invest. 2016 Nov; 96(11):1147-55.

16 Hopp K, Hommerding CJ, Wang X, Ye $\mathrm{H}$, Harris PC, Torres VE. Tolvaptan plus pasireotide shows enhanced efficacy in a PKD1 model. J Am Soc Nephrol. 2015 Jan;26(1):39-47.

17 Flaig SM, Gattone VH, Blazer-Yost BL. Inhibition of cyst growth in PCK and Wpk rat models of polycystic kidney disease with low doses of peroxisome proliferator-activated receptor $\gamma$ agonists. J Transl Int Med. 2016 Sep; $4(3): 118-26$. 